\title{
44580 - EFFECTS OF FIBROMYALGIA PAIN ON COGNITIVE FUNCTION AND QUALITY OF LIFE
}

\author{
Bruce Dick, University of Alberta, Edmonton, AB, Canada; \\ Troy Harker, Dalhousie University; \\ Saifee Rashiq, University of Alberta; \\ $M$ Verrier, University of Alberta;
}

INTRODUCTION: Individuals with FMS experience significant pain and often report significant cognitive dysfunction as a result of pain. However, research on their cognitive performance on attentional tasks has produced mixed results. Unfortunately, these studies did not include measures of other, more basic cognitive processes that might explain and predict these attentional deficits. While it is clear that many individuals who suffer from chronic pain due to FMS experience attentional and other cognitive deficits, the cognitive mechanisms that are affected by their pain are not clear. Further, the effects of these deficits on patients' quality of life (QOL) are also not clear.

METHODS: Local IRB approval was obtained for this study. Thirty Female patients ( $\mathrm{M}$ age: 49.6 years) with chronic pain resulting from Fibromyalgia Syndrome were included in this study. Demographic and general health information were collected along with measures of pain, disability, quality of life, mood, and pain-related fear. Standardized neuropsychological tests and validated computerized paradigms were used to assess attentional performance and working memory capacity. Stepwise linear regression analyses were carried out to ascertain predictors of cognitive disruption and quality of life and the other factors measured in these individuals. RESULTS: Average pain chronicity in this sample was 12.5 years. Patients' overall score of attentional functioning was significantly predicted by their working memory score. Quality of life was significantly predicted by patients' reported level of pain-related disability and their level of pain-related fear. It was not significantly associated with level of cognitive function. SUMMARY: This study of individuals with FMS confirmed previous findings from a general pain clinic population that many individuals with chronic pain experience significant cognitive deficits. Attentional dysfunction was significantly predicted by working memory capacity, highlighting a specific mechanism that may underlie these deficits. This finding suggests that attentional resource capacity plays an important role in specific processes such as selective and sustained attention. Individuals with FMS who had more pain-related disability and who were more fearful of their pain reported poorer QOL. 